\section{每 Heighten Science \\ P U B L I C I T I O N S Corporation ISSN 2575-0186}

\title{
Anatomical Distribution of Intramuscular Lipomas
}

\author{
N K Sferopoulos* \\ Department of Orthopaedics, "G. Gennimatas" Hospital, Thessaloniki, Greece
}

\begin{abstract}
*Address for Correspondence: Dr. N.K.Sferopoulos, Department of Orthopaedics, "G. Gennimatas" Hospital, P. Papageorgiou 3, Thessaloniki, Greece, Tel: 00302310963270; Fax: 00302310968265:

E-mail: sferopoulos@in.gr

Submitted: 04 April 2017

Approved: 17 April 2017

Published: 19 April 2017
\end{abstract}

Copyright: $@ 2017$ Sferopoulos NK. This is an open access article distributed under the Creative Commons Attribution License, which permits unrestricted use, distribution, and reproduction in any medium, provided the original work is properly cited.

\section{EDITORIAL}

Lipomas are the most common type of soft tissue tumor occurring in the subcutaneous tissue. Rarely, lipomas present in the deep soft tissue such as intermuscular, intramuscular, and parosteal sites. When they occur within a skeletal muscle they are called intramuscular lipomas. Intramuscular lipomas may involve both children and adults. They are benign, nontender, deep located, circumscribed but unencapsulated lesions. Intramuscular lipomas account less than $1 \%$ of all lipomas. Most are located within a single muscle (solitary), while cases involving two or more muscles are very rare. They present with typical histological features. They may be divided into the infiltrative, the well-circumscribed and the mixed type. Differential diagnosis of the infiltrative type from liposarcoma is very difficult. Local recurrence may be evident if the surgical margin is not clear. They can occur in almost any anatomical site [1-15].

Intramuscular lipomas of the head may involve the tongue, which is one of the most commonly involved sites [16-24] or the tongue and the submandibular space [25]. They may also involve the cheek [26], the nose [27], the masseter [28] and the temporalis muscle [29,30]. In the eye the orbicularis oculi muscle and the eyelid [31], the superior oblique muscle [32], the superior rectus [33], the medial rectus in a child [34] have been involved.

Intramuscular lipomas of the neck [35] may be localized in the longus colli muscle [36], the platysma [14], the sternocleidomastoid muscle [37-40], the paraspinal muscles [41] and may be retropharyngeal or laryngeal infiltrating [42,43].

The chest wall $[44,45]$ and the intercostal muscles [46] may also be involved. It may appear as a breast mass involving the pectoralis major [47-49] and may also involve the heart; involvement of the left ventricular myocardium, extending to the epicardial space; has also been reported [50]. The large muscles of the trunk may be involved such as the trapezius, the latissimus dorsi, the rhomboid major and the muscles of the anterior abdominal wall $[14,51]$.

Intramuscular lipomas of the limbs may involve the thigh [52-54] and the shoulder [55], which are the most common locations [56-58]. The biceps brachii [59], the subscapularis [60], the deltoid [61-63], the supraspinatous [64,65], the infraspinatus [66] and the brachioradialis [67] may be involved, while localization adjacent to the proximal radius may be complicated by symptomatic radial nerve compression [6870]. Involvement of the tensor fascia lata has also been reported [71] as well as of the vastus lateralis [51] and of the gluteus maximus and gastrocnemius [14]. 
Intramuscular lipomas of the hand and foot are rare. They may be associated with painful wrist triggering with associated carpal tunnel syndrome caused by an intramuscular lipoma of the lumbrical muscle $[72,73]$. They may be localized to the thenar [74-78], in the thenar or hypothenar muscles [79], to the abductor digiti minimi [80], to the extensors [81] and to the flexor hallucis brevis [82].

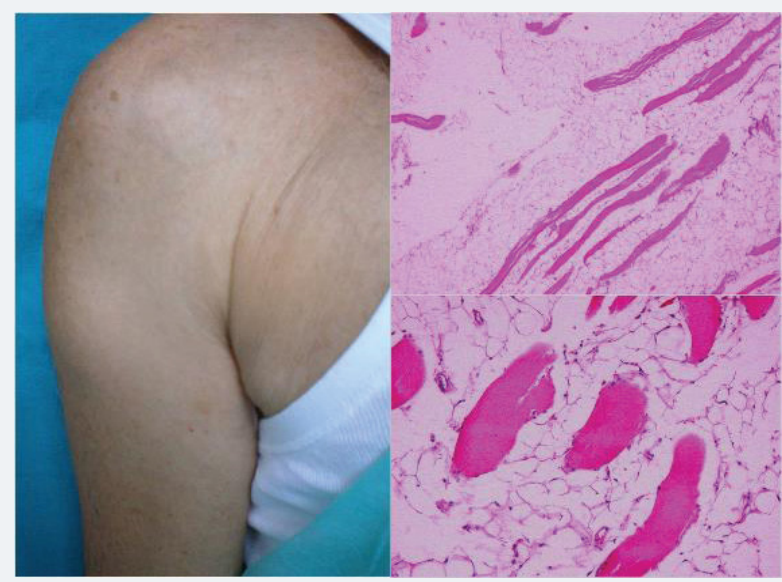

Figure 1: A 65-year-old man with an intramuscular lipoma of the long head of the triceps brachii muscle. Lowpower and high-power views of the tumor showed mature fat cells between and among slightly atrophic skeletal muscle fibers.

\section{REFERENCES}

1. Bennhoff DF, Wood JW. Infiltrating lipomata of the head and neck. Laryngoscope. 1978; 88: 839848. Ref.: https://goo.gl/45orO9

2. Austin RM, Mack GR, Townsend CM, Lack EE. Infiltrating (intramuscular) lipomas and angiolipomas. A clinicopathologic study of six cases. Arch Surg. 1980; 115: 281-284. Ref.: https://goo.gl/AJLW5I

3. Pollack S, Steinfeld AD. Radiographic diagnosis of intramuscular lipoma. R I Med J. 1984; 67: 129130. Ref.: https://goo.gl/Hb1bVf

4. Caprio F, Lanza R, Amoroso L, Manzotti M, Cerioni M, et al. Computed tomographic findings and clinicopathologic features of intramuscular lipoma. Rays. 1985; 10: 39-42. Ref.: https://goo.gl/ILjDZQ

5. Fletcher $C D$, Martin-Bates E. Intramuscular and intermuscular lipoma: neglected diagnoses. Histopathology. 1988; 12: 275-287. Ref.: https://goo.gl/udKU6x

6. Harrington AC, Adnot J, Chesser RS. Infiltrating lipomas of the upper extremities. J Dermatol Surg Oncol 1990; 16: 834-837. Ref.: https://goo.gl/kNvt08

7. Schellong H, Falcone A, Günther M, Schmidt H. [Infiltrating intramuscular lipoma]. Chirurg. 1997; 68: 279-282. Ref.: https://goo.gl/hESRDy

8. Matsumoto $\mathrm{K}$, Hukuda $\mathrm{S}$, Ishizawa $\mathrm{M}$, Chano $\mathrm{T}$, Okabe $\mathrm{H}$. MRI findings in intramuscular lipomas. Skeletal Radiol. 1999; 28: 145-152. Ref.: https://goo.gl/v5cozv

9. Allen B, Rader C, Babigian A. Giant lipomas of the upper extremity. Can J Plast Surg. 2007; 15: 141144. Ref.: https://goo.gl/qHrlkJ

10.Bandeca MC, de Pádua JM, Nadalin MR, Ozório JE, Silva-Sousa YT, et al. Oral soft tissue lipomas: a case series. J Canad Dental Assoc. 2007; 73: 431-434. Ref.: https://goo.gl/MRFWPR

11.Ott I, Sturm K, Losenhausen $\mathrm{H}$, Issing PR. Intramuscular lipoma. A differential diagnosis of great prognostic relevance. HNO. 2009; 57: 1121-1125. Ref.: https://goo.gl/xLWMQ7

12. Su CH, Hung JK, Chang IL. Surgical treatment of intramuscular, infiltrating lipoma. Int Surg 2011; 96 : 56-59. Ref.: https://goo.gl/ela7FR

13.Ramos-Pascua LR, Guerra-Álvarez OA, Sánchez-Herráez S, Izquierdo-García FM, MaderueloFernández JÁ. Intramuscular lipomas: Large and deep benign lumps not to underestimated. 
Review of a series of 51 cases. Rev Esp Cir Ortop Traumatol. 2013; 57: 391-397. Ref.: https://goo.gl/93THgM

14. Han HH, Choi JY, Seo BF, Moon SH, Oh DY, et al. Treatment for intramuscular lipoma frequently confused with sarcoma: a 6 year restrospectivestudy and literature review. Biomed Res Int. 2014. Ref.: https://goo.gl/JKAB4u

15. McTighe S, Chernev I. Intramuscular lipoma: a review of the literature. Orthop Rev (Pavia). 2014; 6 : 5618. Ref.: https://goo.gl/1hK39Q

16.Garavaglia J, Gnepp D. Intramuscular (infiltrating) lipoma of the tongue. Oral Surg Oral Med Oral Pathol. 1987; 63: 348-350. Ref.: https://goo.gl/g5jusy

17. Takeda Y. Intramuscular lipoma of the tongue: report of a rare case. Ann Dent. 1989; 48: 22-24. Ref.: https://goo.gl/7SIFg5

18. Keskin G, Ustundag E, Ercin C. Multiple infiltrating lipomas of the tongue. J Laryngol Otol. 2002; 116 : 395-397. Ref.: https://goo.gl/B9Gu5k

19. Collela G, Lanza A, Rossiello L, Rossiello R. Infiltrating lipoma of the tongue. Oral Oncology Extra. 2004; 40: 33-35. Ref.: https://goo.gl/60M7n9

20.Akbulut M, Aksoy A, Bir F. Intramuscular lipoma of the tongue: a case report and review of the literature. Aegean Pathol. 2005; 2: 146-9. Ref.: https://goo.gl/qjzgia

21.Chung JC, Ng RW. A huge tongue lipoma. Otolaryngol Head Neck Surg. 2007; 137: 830-831. Ref.: https://goo.gl/USxjOl

22. Colella G, Biondi $P$, Caltabiano $R$, Vecchio GM, Amico $P$, et al. Giant intramuscular lipoma of the tongue: a case report and literature review. Cases J. 2009; 2: 7906. Ref.: https://goo.gl/J24yj5

23.Garg M, Aggarwal R, Rajeev S. Intramuscular lipoma of tongue. J Cutan Aesthet Surg. 2011; 4: 152 153. Ref.: https://goo.gl/X9xV8i

24.Amirzedeh A, Klaustermeyer W. Intramuscular lipoma of the tongue masquerading as angioedema. Ear, Nose Throat J. 2013; 92: E4-E5. Ref.: https://goo.gl/Z6jQ08

25. Dattilo DJ, Ige JT, Nwana EJ. Intraoral lipoma of the tongue and submandibular space: report of a case. J Oral Maxillofac Surg. 1996; 54: 915-917. Ref.: https://goo.gl/j0dCmP

26. Piattelli A, Fioroni M, Rubini C. Intramuscular lipoma of the cheek: a case report. J Oral Maxillofac Surg. 2000; 58: 817-819. Ref.: https://goo.gl/PclYJX

27.Vincent J, Baker P, Grischkan J, Fernandez Faith E. Subcutaneous midline nasal mass in an infant due to an intramuscular lipoma. Pediatr Dermatol. 2017. Ref.: https://goo.gl/FZley9

28. Tsumuraya G, Yamada $H$, Shimizu H, Hamada $Y$. Intramuscular lipoma in the masseter muscle: a case report. Br J Oral Maxillofac Surg. 2014; 52: e21-e23. Ref.: https://goo.gl/OjsN5A

29.Uemura T, Suse T, Yokoyama T, Mitsukawa N, Yoshikawa A. Intramuscular benign lipoma of the temporalis muscle. Scand J Plast Reconstr Surg Hand Surg. 2002; 36: 231-234. Ref.: https://goo.gl/OXGcYf

30.Ban M, Kitajima Y. Intramuscular lipoma within the temporal muscle. Int J Dermatol. 2002; 41: 689 690. Ref.: https://goo.gl/mDFW00

31. Charles NC, Palu RN. Intramuscular lipoma of the eyelid. Ophthalmic Surg Lasers. 2000; 31: 340341. Ref.: https://goo.gl/HkxLza

32.Dutton J, Wright J. Intramuscular lipoma of the superior oblique muscle. Orbit. 2006; 25: $227-233$. Ref.: https://goo.gl/bIEvRT

33. Hristodulopulos V, Medel R. Intramuscular lipoma of superior rectus muscle. Ophthal Plast Reconstr Surg. 2016; 32: e52-e55. Ref.: https://goo.gl/E88v0k

34. Shiraki K, Kamo M, Sai T, Kamo R. Rare site for an intramuscular lipoma. Lancet. 2002; 359: 2077. Ref.: https://goo.gl/E5PqSU

35.Lerosey Y, Choussy O, Gruyer X, François A, Marie JP, et al. Infiltrating lipoma of the head and neck: a report of one pediatric case. Int J Pediatr Otorhinolaryngol. 1999; 47: 91-95. Ref.: https://goo.gl/cUvk54

36. Pichierri A, Marotta N, Raco A, Delfini R. Intramuscular infiltrating lipoma of the longus colli muscle: a very rare cause of neck structures compression. Cent Eur Neurosurg. 2010; 71: 157-159. Ref.: https://goo.gl/xtwfqj 
37. Mattel SF, Persky MS. Infiltrating lipoma of the sternocleidomastoid muscle. Laryngoscope 1983; 93: 205-207. Ref.: https://goo.gl/XRPbEV

38. Moumoulidis I, Durvasula $P$, Jani $P$. Well-circumscribed intramuscular lipoma of the sternocleidomastoid muscle. Auris Nasus Larynx. 2004; 31: 283-285. Ref.: https://goo.gl/AoYRQ0

39.Ozcan C, Gorur K, Talas D, Aydin O. Intramuscular benign lipoma of the sternocleidomastoid muscle: a rare cause of neck mass. Eur Arch Otorhinolaryngol. 2005; 262: 148-150. Ref.: https://goo.gl/cq3chV

40.Sohn W, Kim JH, Jung SN, Kwon H, Cho KJ. Intramuscular lipoma of the sternocleidomastoid muscle. J Craniofacial Surg. 2010; 21: 1976-1978. Ref.: https://goo.gl/XdMKNb

41.Dattolo RA, Nesbit GM, Kelly KE, Cupp CL. Infiltrating intramuscular lipoma of the paraspinal muscles. Ann Otol Rhinol Laryngol. 1995; 104: 582-584. Ref.: https://goo.gl/zqOgQ0

42.Senchenkov A, Werning J, Staren E. Radiographic assessment of the infiltrating retropharyngeal lipoma. Otolaryngol Head Neck Surg. 2001; 125: 658-660. Ref.: https://goo.gl/da7kQ3

43.Deschler DG, Lee K, Tami TA. Laryngeal infiltrating intramuscular lipoma. Otolaryngol Head Neck Surg. 1993; 108: 374-377. Ref.: https://goo.gl/s3F6HK

44.Takamori S. Miwa K. Hayashi A, Shirouzu K. Intramuscular lipoma in the chest wall. Eur J Cardiothorac Surg. 2004; 26: 1038. Ref.: https://goo.gl/AWOfdz

45.Lee JH, Do HD, Lee JC. Well-circumscribed type of intramuscular lipoma in the chest wall. J Cardiothorac Surg. 2013; 8: 181. Ref.: https://goo.gl/DtFCqE

46. Hwang J, Jo WM, Min BJ, Shin JS. Deep-seated intramuscular lipoma penetrates the intercostal muscle. J Thorac Dis. 2015; 7: E493-E495. Ref.: https://goo.gl/Mkm2BS

47.Gopal U, Patel MH, Wadhwa MK. Intramuscular lipoma of the pectoralis major muscle. J Postgraduate Med. 2002; 48: 330-331. Ref.: https://goo.gl/VP7V4B

48. Pant R, Poh ACC, Hwang SG. An unusual case of an intramuscular lipoma of the pectoralis major muscle simulating a malignant breast mass. Annals Academy of Medecine Singapore. 2005; 34 275-276. Ref.: https://goo.gl/Q2XzC9

49. D'Alfonso TM, Shin SJ. Intramuscular lipoma arising within the pectoralis major muscle presenting as a radiographically detected breast mass. Arch Pathol Lab Med. 2011; 135: 1061-1063. Ref.: https://goo.gl/uvkeCU

50. Oyama N, Oyama N, Komatsu H, Okita K, Yonezawa K, et al. Left ventricular asynchrony caused by an intramuscular lipoma: computed tomographic and magnetic resonance detection. Circulation. 2003; 107: e200-e2001. Ref.: https://goo.gl/DwEMOg

51.Kindblom LG, Angervall L, Stener B, Wickbom I. Intermuscular and intramuscular lipomas and hibernomas. A clinical, roentgenologic, histologic, and prognostic study of 46 cases. Cancer. 1974 33: 754-762. Ref.: https://goo.gl/FyWSPj

52.Bjerregaard $\mathrm{P}$, Hagen $\mathrm{K}$, Daugaard S, Kofoed $\mathrm{H}$. Intramuscular lipoma of the lower limb. Long-term follow-up after local resection. J Bone Joint Surg Br. 1989; 71: 812-815. Ref.: https://goo.gl/hIP8le

53.Gutknecht DR. Painful intramuscular lipoma of the thigh. South Med J. 2004; 97: 1121-1122. Ref.: https://goo.gl/UixNvt

54.Ezirmik N, Yildiz K. Deep intramuscular lipoma in thigh. Med J Bakirkoy. 2011; 7: 167-169.

55.Grandbois L, Vade A, Lim-Dunham J, Al-Masri H. MRI findings of an intermuscular lipoma in a 2-yearold. Pediatr Radiol. 2006; 36: 974-976. Ref.: https://goo.gl/X8iVSV

56. Kransdorf MJ, Bancroft LW, Peterson JJ, Murphey MD, Foster WC, et al. Imaging of fatty tumors: distinction of lipoma and well-differentiated liposarcoma. Radiology. 2002; 224: 99-104. Ref.: https://goo.gl/k3DgcW

57.Bassett MD, Schuetze SM, Disteche C, Norwood TH, Swisshelm K, et al. Deep-seated, well differentiated lipomatous tumors of the chest wall and extremities. The role of cytogenetics in classification and prognostication. Cancer. 2005; 103: 409-416. Ref.: https://goo.gl/6cVVKA

58.Nishida J, Morita T, Ogose A, Okada K, Kakizaki H, et al. Imaging characteristics of deep-seated lipomatous tumors: intramuscular lipoma, intermuscular lipoma, and lipoma-like liposarcoma. J Orthop Sci. 2007; 12: 533-541. Ref.: https://goo.gl/2pG6Ap

59.Lahrach K, el Kadi KI, Mezzani A, Marzouki A, Boutayeb F. An unusual case of an intramuscular lipoma of the biceps brachii. Pan African Med J. 2013; 15: 40. Ref.: https://goo.gl/2VHSgu 
60.Balabram D, Cabral CC, Filho OdeP, Barros CP. Intramuscular lipoma of the subscapularis muscle. Sao Paulo Med J. 2014; 132: 65-67. Ref.: https://goo.gl/YH22uD

61. Warner JJ, Madsen N, Gerber C. Intramuscular lipoma of the deltoid causing shoulder pain: report of two cases. Clin Orthop Relat Res. 1990; 253: 110-112. Ref.: https://goo.gl/vdC7jm

62. Ramos Pascua L, Alonso León A, Santos Sánchez JA, Ferrández Portal L. Intramuscular lipoma of the deltoid mimicking a sarcoma. A case report. Chir Organi Mov. 2001; 86: 153-157. Ref.: https://goo.gl/DxtQvg

63. Kapetanakis S, Papathanasiou J, Dermon A, Dimitrakopoulou A, Ververidis A, et al. Unusual intramuscular lipoma of the deltoid muscle. Folia Med. 2010; 52: 68-71. Ref.: https://goo.gl/3w1kx5

64.Egea Martinez JM, Mena JF. Lipoma of the supraspinatus muscle causing impingement syndrome a case report. J Shoulder Elbow Surg. 2009; 18: 3-5. Ref.: https://goo.gl/nLNTVG

65.Ferrari L, Haynes P, Mack J, DiFelice GS. Intramuscular lipoma of the supraspinatus causing impingement syndrome. Orthopedics 2009; 32: 601. Ref.: https://goo.gl/3AwqjK

66. Sungur N, Kilinç H, Ozdemr R, Sensöz O. An infiltrating intramuscular lipoma of the brachioradialis muscle. Ann Plast Surg. 2001; 46: 353-354. Ref.: https://goo.gl/2GqsxT

67. Coraci D, Santilli V, Padua L. Painful Intramuscular Lipoma of the Infraspinatus: Unusual Location and Presentation. Orthopedics. 2016; 39: 206. Ref.: https://goo.gl/B8eyVO

68. Cappellini O. A case of dissociated paralysis of the radial nerve caused by intramuscular lipoma. Chir Organi Mov. 1958; 45: 338-343. Ref.: https://goo.gl/CMvl0k

69. Manes E. On a case of dissociated paralysis of the radial nerve caused by intramuscular lipoma. Osp Ital Chir. 1968; 19: 165-172. Ref.: https://goo.gl/OdNYnB

70.Allagui M, Maghrebi S, Touati B, Koubaa M, Hadhri R, et al. Posterior interosseous nerve syndrome due to intramuscular lipoma. Eur Orthop Traumatol. 2014; 5: 75-79. Ref.: https://goo.gl/oO4eie

71.Feng A, Igolnikov I, Brown CH, Weinik MM. Poster 197-EHip Pain Secondary to Intramuscular Lipoma of Tensor Fascia Lata: A Case Report. PM R. 2016; 8: S329-S330. Ref.: https://goo.gl/ct6reN

72. Cossey AJ, Stranks GJ. Intramuscular lipoma in an anomalous muscle belly of the middle finger lumbrical as a cause of carpal tunnel syndrome and trigger wrist. Orthopedics. 2003; 26: 85-86. Ref.: https://goo.gl/xAeGSO

73.Berlund $P$, Kalamaras $M$. A case report of trigger wrist associated with carpal tunnel syndrome caused by an intramuscular lipoma. Hand Surg. 2014; 19: 237-239. Ref.: https://goo.gl/yUtJ5Z

74.Aldredge WM, Halpert B. Lipoma of the thenar. Surgery. 1948; 24: 853. Ref.: https://goo.gl/Xpx8hs

75.Zamora MA, Zamora CA, Samayoa EA, Morales HA, Ceballos JF. High-resolution ultrasonography in an aggressive thenar intramuscular lipoma. J Ultrasound Med. 2005; 24: 1151-1155. Ref.: https://goo.gl/9sCYkU

76. Mohan L, Semoes J. Thenar intramuscular lipoma: an unusual case. Internet J Surg. 2008; 17:1-3. Ref.: https://goo.gl/JI17JE

77.Chernev I, Tingey S. Thenar intramuscular lipoma: A case report. J Med Cases. 2013; 4: 676-678. Ref.: https://goo.gl/XGljfg

78. Papakostas T, Tsovilis AE, Pakos EE. Intramuscular lipoma of the thenar: A Rare Case. Arch Bone Jt Surg. 2016; 4: 80-82. Ref.: https://goo.gl/cwB00v

79. Lee YH, Jung JM, Baek GH, Chung MS. Intramuscular lipoma in thenar or hypothenar muscles. Hand Surg. 2004; 9: 49-54. Ref.: https://goo.gl/EdOM1t

80.Lui TH. Intramuscular lipoma of abductor digiti minimi mimicking intramuscular haemangioma. BMJ Case Rep. 2013. Ref.: https://goo.gl/f0nDgL

81.Rispoli L, Singh JR, Piesco J. Unusual location of intramuscular lipoma presenting as an extensor tendon tear: A diagnostic dilemma. Am J Phys Med Rehabil. 2017. Ref.: https://goo.gl/opvxXy

82. Kalmar G, Doobay N. Intramuscular lipoma of the flexor hallucis brevis muscle. A case report. J Am Podiatr Med Assoc. 2017; 107: 80-84. Ref.: https://goo.gl/ftTLjS 